\title{
STARK WIDTHS OF Sc IV SPECTRAL LINES WITHIN 4s-4p TRAN- SITION ARRAY
}

\author{
MILAN S. DIMITRIJEVIĆC $1,2, \star$ \\ ${ }^{1}$ Astronomical Observatory, Belgrade, Serbia \\ ${ }^{2}$ LERMA, Observatoire de Paris, PSL Research University, CNRS, Sorbonne Universities, UPMC Univ. Paris, France
}

\begin{abstract}
Stark full widths at half maximum of six $4 \mathrm{~s}$ - 4p Sc IV spectral lines, broadened by collisions with electrons, have been calculated for electron density of $10^{17} \mathrm{~cm}^{-3}$ by using the modified semiempirical method. The results are provided for temperatures from $10000 \mathrm{~K}$ to $160000 \mathrm{~K}$. They are used also to discuss the similarities of Sc IV spectral lines within multiplet, supermultiplet and transition array. Obtained results will be included in the STARK-B database which is also a part of Virtual atomic and molecular data center(VAMDC).
\end{abstract}

Keywords: Stark widths, spectral lines, line profiles, Sc IV.

\section{INTRODUCTION}

Broadening of spectral lines by fluctuating electric microfield produced by charged particles moving near an emitter/absorber, known as Stark broadening, is important for many topics in physics and astronomy. Data on Stark broadening are needed for laboratory plasmas diagnostic and investigation (Konjević, 1999; Torres et al., 2006; Peláez et al., 2009), laser produced plasma analysis, modelling and diagnostics (Richou \& Molitor, 1970; Gornushkin et al., 1999; Sorge et al., 2000; Seidel et al., 2001) and inertial fusion plasma (Keane et al., 1990; Griem, 1992). Stark broadening data are also useful in technology, as for example for laser welding and piercing of various metals (Dimitrijević \& Sahal-Bréchot, 2014), design, investigation and improvement of effectivity of various light sources based on plasma (Wieser et al., 1997; Seidel et al., 2001), and the design and developing of lasers (Csillag \& Dimitrijević, 2004; Dimitrijević \& SahalBréchot, 2014). In many astrophysical plasmas Stark broadening of spectral lines is very important or at least non negligible and should be taken into account (Beauchamp et al., 1997; Popović et al., 2001; Dimitrijević, 2003; Dimitrijević \& Sahal-Bréchot, 2014).

Stark broadening data are particularly important for stellar plasma research, and for analysis and synthesis of stellar spectra (Beauchamp et al., 1997; Dimitrijević \& Sahal-Bréchot, 2014). In atmospheres of white dwarfs, pre white dwarf stars, and post AGB(Asymptotic Giant Branch) stars this is usually the main broadening mechanism (Tankosić et al., 2003; Milovanović et al., 2004; Simić et al., 2006; Dufour et al., 2011). It can not be neglected and in some atmospheric layers of A and late B stars (Simić et al., 2005b,a, 2009). As an example, (Popović et al., 2001) have shown that, for A-type star atmospheres, the inclusion of Stark broadening can change the equivalent widths by $10-45 \%$.
Consequently, for determination of abundances, the importance of Stark broadening of analysed spectral lines should be checked.

For some astrophysical problems, like modelling of stellar atmospheres, radiative transfer or derivation of accurate atmospheric parameters, we need a very large number of various atomic data, including Stark broadening (Dufour et al., 2011). We note as well that (Rauch et al., 2007) emphasized the crucial importance of an accurate and as much as possible complete Stark broadening data set for large number of atoms and ions, "for sophisticated analysis of stellar spectra by means of NLTE model atmospheres".

The development of satellite-born astronomy, enabling to obtain stellar spectra with earlier not possible resolution, as well as development of computers enabling very sophisticated NLTE synthesis and modelling of stellar spectra, made that earlier astrophysically insignificant data on trace elements now are important. Scandium is present and observed in stellar spectra (Adelman \& Pintaldo, 2000; Adelman et al., 2001; Kahraman Alicavus et al., 2017) so that its Stark broadening parameters are needed. However, there is no at all experimental data for Stark broadening of scandium lines. For Sc IV there is no neither theoretical data. Results of Stark broadening parameter calculations exist only for Sc II (Popović \& Dimitrijević, 1996, 1997), Sc III (Dimitrijević \& Sahal-Bréchot, 1992), Sc X (Dimitrijević \& Sahal-Bréchot, 1998a,b; Elabidi \& Sahal-Bréchot, 2011) and Sc XI (Dimitrijević \& Sahal-Bréchot, 1998a,b).

In order to provide the Stark broadening data, needed first of all for stellar astrophysics, for Sc IV spectral lines, which are absent completely in the literature, Stark Full Widths at Half intensity Maximum(FWHM) $W$ for six transitions have been calculated here, by using the modified semiempirical method (MSE, Dimitrijević \& Konjević (1980); Dimitrijević \& Kršljanin (1986); Dimitrijević \& Popović (2001)) for collisions of Sc IV ions with electrons. The obtained results will be used for the discussion of simi-

\footnotetext{
^ Corresponding author: mdimitrijevic@aob.rs
} 
larities of Stark widths within a supermultiplet. Also, the obtained results will be implemented in the STARK-B database (SahalBréchot et al., 2015, 2017), a repository for spectral line broadening and shifts due to collisions with charged particles, and also a node of Virtual Atomic and Molecular Data Center - VAMDC (?Rixon et al., 2011; Dubernet et al., 2016).

\section{THE MODIFIED SEMIEMPIRICAL METHOD}

Within the frame of the modified semiempirical(MSE) approach (Dimitrijević \& Konjević, 1980; Dimitrijević \& Kršljanin, 1986; Popović et al., 2001) the electron impact full width at half intensity maximum(FHWM) of an isolated non hydrogenic ion line is given as

I

$$
\begin{gathered}
W_{M S E}=N \frac{8 \pi}{3} \hbar^{22}\left(\frac{2 m}{)}\right)^{1 / 2} \frac{\pi}{\sqrt{3}} \frac{\lambda^{2}}{2 \pi c} \times \\
\times\left\{\sum_{\ell_{i} \pm 1} \sum_{L_{i^{\prime}} J_{i^{\prime}}} \mathfrak{R}^{2}\left[n_{i} \ell_{i} L_{i} J_{i}, n_{i}\left(\ell_{i} \pm 1\right) L_{i^{\prime}} J_{i^{\prime}}\right] \widetilde{g}\left(x_{\ell_{i}, \ell_{i} \pm 1}\right)+\right. \\
+\sum_{\ell_{f} \pm 1} \sum_{L_{f^{\prime}} J_{f^{\prime}}} \mathfrak{R}^{2}\left[n_{f} \ell_{f} L_{f} J_{f}, n_{f}\left(\ell_{f} \pm 1\right) L_{f^{\prime}} J_{f^{\prime}}\right] \widetilde{g}\left(x_{\ell_{f}, \ell_{f} \pm 1}\right)+ \\
\left.+\left(\sum_{i^{\prime}} \mathfrak{R}_{i i^{\prime}}^{2}\right)_{\Delta n \neq 0} g\left(x_{n_{i}, n_{i}+1}\right)+\left(\sum_{f^{\prime}} \mathfrak{R}_{f f^{\prime}}^{2}\right)_{\Delta n \neq 0} g\left(x_{n_{f}, n_{f}+1}\right)\right\},
\end{gathered}
$$

where the initial level is denoted as $i$ and the final one as $f$. The square of the matrix element $\left\{\mathfrak{R}^{2}\left[n_{k} \ell_{k} L_{k} J_{k},\left(\ell_{k} \pm 1\right) L_{k^{\prime}} J_{k^{\prime}}\right], \quad k=\right.$ $i, f\}$ is given by the expression

$$
\begin{gathered}
\mathfrak{R}^{2}\left[n_{k} \ell_{k} L_{k} J_{k}, n_{k}\left(\ell_{k} \pm 1\right) L_{k}^{\prime} J_{k}^{\prime}\right]= \\
=\frac{\ell_{>}}{2 J_{k}+1} Q\left[\ell_{k} L_{k},\left(\ell_{k} \pm 1\right) L_{k}^{\prime}\right] Q\left(J_{k}, J_{k}^{\prime}\right)\left[R_{n_{k}^{*} \ell_{k}^{*}}^{n_{k}^{*}\left(\ell_{k} \pm 1\right)}\right]^{2} .
\end{gathered}
$$

Here, $\ell_{>}=\max \left(\ell_{k}, \ell_{k} \pm 1\right)$ and

$$
\left(\sum_{k^{\prime}} \mathfrak{R}_{k k^{\prime}}^{2}\right)_{\Delta n \neq 0}=\left(\frac{3 n_{k}^{*}}{2 Z}\right)^{2} \frac{1}{9}\left(n_{k}^{* 2}+3 \ell_{k}^{2}+3 \ell_{k}+11\right)
$$

In Eq.(1)

$$
x_{\ell_{k}, \ell_{k^{\prime}}}=\frac{E}{\Delta E_{\ell_{k}, \ell_{k^{\prime}}}}, \quad k=i, f
$$

$E=\frac{3}{2} k T$ is the electron kinetic energy and $\Delta E_{\ell_{k}, \ell_{k^{\prime}}}=\left|E_{\ell_{k}}-E_{\ell_{k^{\prime}}}\right|$ is the energy difference between levels $\ell_{k}$ and $\ell_{k} \pm 1(k=i, f)$,

$$
x_{n_{k}, n_{k}+1} \approx \frac{E}{\Delta E_{n_{k}, n_{k}+1}},
$$

where for $\Delta n \neq 0$, the energy difference between energy levels with $n_{k}$ and $n_{k}+1, \Delta E_{n_{k}, n_{k}+1}$ is approximated as

$$
\Delta E_{n_{k}, n_{k}+1}=2 Z^{2} E_{H} / n_{k}^{* 3}
$$

$n_{k}^{*}=\left[E_{H} Z^{2} /\left(E_{i o n}-E_{k}\right)\right]^{1 / 2}$ is the effective principal quantum number, $Z$ is the residual ionic charge(charge "seen" by optical electron; for example $Z=1$ for neutrals) and $E_{\text {ion }}$ is the appropriate spectral series limit. $N$ and $T$ are electron density and temperature, respectively and $Q\left(\ell L, \ell^{\prime} L^{\prime}\right), Q\left(J, J^{\prime}\right)$ multiplet and line factors. With $g(x)(\mathrm{Griem}, 1968,1974)$ and $\widetilde{g}(x)$ (Dimitrijević \& Konjević, 1980) are denoted the corresponding Gaunt factors. The needed radial integrals $\left[R_{n_{k}^{*} \ell_{k}}^{n_{k}^{*} \ell_{k} \pm 1}\right]$ have been calculated here within the Coulomb approximation by using the method of (Bates \& Damgaard, 1949) and the tables of (Oertel \& Shomo, 1968). We note that if for the higher principal quantum numbers there is no the corresponding data in (Oertel \& Shomo, 1968), the radial integrals may be calculated with the help of the article of (Van Regemorter et al., 1979).

\section{RESULTS AND DISCUSSION}

Stark widths(FWHM) of six Sc IV spectral lines, broadened by collisions with electrons, have been calculated using Eqs.(1-4) wihin the frame of MSE method (Dimitrijević \& Konjević, 1980; Popović et al., 2001). Energy levels and ionization energy needed for their calculations have been taken from (Sugar \& Corliss, 2004).

In Table 1, the results of our MSE calculations of Stark widths(FWHM) for six spectral lines broadened by electronimpacts, for a perturber density of $10^{17} \mathrm{~cm}^{-3}$ and for a set of temperatures from $10000 \mathrm{~K}$ to $160000 \mathrm{~K}$, are shown. The chosen temperature range is of interest in astrophysics, laboratory plasma, fusion research, technology and for lasers and laser produced plasma. If one needs perturber densities lower than $10^{17} \mathrm{~cm}^{-3}$ the extrapolation is linear. For higher perturber densities extrapolation is linear if the influence of Debye screening is negligible or reasonably small. In Table 1 are also given the observed wavelength and the multiplet number from the NIST database (Kramida et al., 2017). It is shown as well the quantity $3 k T / 2 \Delta E$, representing the ratio of the average energy of free electrons, $E=3 k T / 2$, and the energy difference of initial or final and the closest perturbing level, $\Delta E$. It is calculated for $T=20000 \mathrm{~K}$ in the following way:

$$
\Delta E=\operatorname{Max}\left[E / \Delta E_{i, i^{\prime}}, E / \Delta E_{f, f^{\prime}}, \Delta E_{n_{i}, n_{i}+1}, \Delta E_{n_{f}, n_{f}+1}\right]
$$

This ratio shows which collisions are dominant. $3 k T / 2 \Delta E=$ 1 is the threshold for the corresponding inelastic transition. If it is lower than one, elastic collisions are dominant and it is so called low temperature limit. If it is large, say larger than 50, high temperature limit approximation can be applied.

The calculated triplets belong to the same $4 \mathrm{~s}^{3} \mathrm{P}^{o}-4 \mathrm{p}^{3} \mathrm{~S}$ multiplet, singlets to the same $4 \mathrm{~s}^{1} \mathrm{P}^{o}-4 \mathrm{p}^{1} \mathrm{~L}(\mathrm{~L}=\mathrm{S}, \mathrm{P}, \mathrm{D})$ supermultiplet and all calculated transitions to the same, $4 \mathrm{~s}-4 \mathrm{p}$ transition array. (Wiese \& Konjević, 1982) concluded after examination of regularities and similarities in plasma broadened spectral line widths that line widths in angular frequency units in multiplets usually agree within a few per cent, in supermultiple within about 30 per 
Table 1. This table gives electron-impact broadening(Stark broadening) Full Widths at Half Intensity Maximum(W) for Sc IV spectral lines, for a perturber density of $10^{17} \mathrm{~cm}^{-3}$ and temperatures from 10000 to $160000 \mathrm{~K}$. Also are given multiplet numbers and wavelengths from NIST database (Kramida et al., 2017) and $3 k T / 2 \Delta E$ for $T=20000 \mathrm{~K}$, where $\Delta E$ is the energy difference between closest perturbing level and the closer of initial and final levels.

\begin{tabular}{|c|c|c|c|}
\hline Transition & $\mathrm{T}(\mathrm{K})$ & $\mathrm{W}[\AA]$ & $\mathrm{W}\left[10^{12} \mathrm{~s}^{-1}\right]$ \\
\hline \multirow{2}{*}{$\begin{array}{c}\text { Sc IV } 3 s^{2} 3 p^{5} 4 s^{1} P^{o}-3 s^{2} 3 p^{5} 4 p^{1} P \\
\text { (Mult. } 100)\end{array}$} & 10000. & 0.0735 & 0.294 \\
\hline & 20000. & 0.0519 & 0.208 \\
\hline $2171.2 \AA$ & 40000. & 0.0367 & 0.147 \\
\hline \multirow{2}{*}{$3 \mathrm{kT} / 2 \Delta \mathrm{E}=0.541$} & 80000 . & 0.0260 & 0.104 \\
\hline & 160000. & 0.0194 & 0.0776 \\
\hline \multirow{5}{*}{$\begin{array}{c}\text { Sc IV } 3 \mathrm{~s}^{2} 3 \mathrm{p}^{5} 4 \mathrm{~s}^{1} \mathrm{P}^{o}-3 \mathrm{~s}^{2} 3 \mathrm{p}^{5} 4 \mathrm{p}^{1} \mathrm{D} \\
\text { (Mult. 101) } \\
2119.0 \AA \\
3 \mathrm{kT} / 2 \Delta \mathrm{E}=0.526\end{array}$} & 10000. & 0.0802 & 0.337 \\
\hline & 20000. & 0.0621 & 0.260 \\
\hline & 40000 . & 0.0479 & 0.201 \\
\hline & 80000 . & 0.0399 & 0.167 \\
\hline & 160000. & 0.0344 & 0.144 \\
\hline \multirow{5}{*}{$\begin{array}{c}\text { Sc IV } 3 \mathrm{~s}^{2} 3 \mathrm{p}^{5} 4 \mathrm{~s}^{1} \mathrm{P}^{o}-3 \mathrm{~s}^{2} 3 \mathrm{p}^{5} 4 \mathrm{p}^{1} \mathrm{~S} \\
\text { (Mult. 102) } \\
1665.9 \AA \\
3 \mathrm{kT} / 2 \Delta \mathrm{E}=0.453\end{array}$} & 10000. & 0.0472 & 0.321 \\
\hline & 20000. & 0.0334 & 0.227 \\
\hline & 40000. & 0.0240 & 0.163 \\
\hline & 80000. & 0.0180 & 0.122 \\
\hline & 160000. & 0.0146 & 0.0989 \\
\hline \multirow{5}{*}{$\begin{array}{c}\text { Sc IV } 3 \mathrm{~s}^{2} 3 \mathrm{p}^{5} 4 \mathrm{~s}^{3} \mathrm{P}_{2}^{o}-3 \mathrm{~s}^{2} 3 \mathrm{p}^{5} 4 \mathrm{p}^{3} \mathrm{~S}_{1} \\
\text { (Mult. 85) } \\
2586.9 \AA \\
3 \mathrm{kT} / 2 \Delta \mathrm{E}=0.606\end{array}$} & 10000. & 0.0964 & 0.271 \\
\hline & 20000. & 0.0682 & 0.192 \\
\hline & 40000 . & 0.0482 & 0.136 \\
\hline & 80000. & 0.0344 & 0.0969 \\
\hline & 160000. & 0.0272 & 0.0765 \\
\hline \multirow{5}{*}{$\begin{array}{c}\text { Sc IV } 3 \mathrm{~s}^{2} 3 \mathrm{p}^{5} 4 \mathrm{~s}^{3} \mathrm{P}_{1}^{o}-3 \mathrm{~s}^{2} 3 \mathrm{p}^{5} 4 \mathrm{p}^{3} \mathrm{~S}_{1} \\
\text { (Mult. 85) } \\
2678.0 \AA \\
3 \mathrm{kT} / 2 \Delta \mathrm{E}=0.606\end{array}$} & 10000. & 0.104 & 0.274 \\
\hline & 20000. & 0.0737 & 0.194 \\
\hline & 40000 . & 0.0521 & 0.137 \\
\hline & 80000. & 0.0373 & 0.0980 \\
\hline & 160000. & 0.0295 & 0.0774 \\
\hline \multirow{5}{*}{$\begin{array}{c}\text { Sc IV } 3 \mathrm{~s}^{2} 3 \mathrm{p}^{5} 4 \mathrm{~s}^{3} \mathrm{P}_{0}^{o}-3 \mathrm{~s}^{2} 3 \mathrm{p}^{5} 4 \mathrm{p}^{3} \mathrm{~S}_{1} \\
\text { (Mult. 85) } \\
2906.5 \AA \\
3 \mathrm{kT} / 2 \Delta \mathrm{E}=0.606\end{array}$} & 10000. & 0.125 & 0.279 \\
\hline & 20000. & 0.0886 & 0.197 \\
\hline & 40000. & 0.0626 & 0.140 \\
\hline & 80000 . & 0.0451 & 0.101 \\
\hline & 160000. & 0.0357 & 0.0797 \\
\hline
\end{tabular}

cent and within a transition array within about 40 per cent. The obtained here results give us an opportunity to check the similarities of Stark line widths within multiplet, supermultiplet and transition array for Sc IV in order to see if they could be used for derrivation of missing values. The transformation of the Stark widths expressed in $\AA$-units to the widths in angular frequency units may be performed by the following formula:

$$
W(\AA)=\frac{\lambda^{2}}{2 \pi c} W\left(s^{-1}\right),
$$

where $c$ is the speed of light.

From Table 1 we obtained that within the Sc IV $4 \mathrm{~s}^{3} \mathrm{P}^{o}$ $4 \mathrm{p}^{3} \mathrm{~S}$ multiplet the smalest Stark width value is $2.87 \%$ smaller from largest at $T=10000 \mathrm{~K}$ and $4.02 \%$ at $T=160000 \mathrm{~K}$. Within Sc IV $4 \mathrm{~s}^{1} \mathrm{P}^{o}-4 \mathrm{p}^{1} \mathrm{~L}(\mathrm{~L}=\mathrm{S}, \mathrm{P}, \mathrm{D})$ supermultiplet, the smallest $\mathrm{W}$ is $12.76 \%$ smaller from the largest one at $T=10000 \mathrm{~K}$ and $46.11 \%$ at
$T=160000 \mathrm{~K}$. For $4 \mathrm{~s}-4 \mathrm{p}$ transition array these values are $19.58 \%$ and $46.88 \%$. We can conclude that in average, these values are in agreement with (Wiese \& Konjević, 1982) conclusions and that disagreement increases with the increase of temperature.

If we have Stark width value for one member of multiplet, supermultiplet or transition array, the needed width for another member, can be obtained with the help of the expression:

$$
W_{1}=\left(\frac{\lambda_{1}}{\lambda}\right)^{2} W
$$

Here, with $W_{1}$ is denoted the corrected width, for the spectral line with the wavelength $\lambda_{1}$, and $\lambda$ is the wavelength of the line for which we have the Stark width value $W$.

The Stark FWHM for Sc IV spectral lines obtained within the modified semiempirical method and shown in Table 1, will be also implemented in the STARK-B database (Sahal-Bréchot et al., 
2015,2017 ), created first of all for the investigations, modelling and diagnostics of the plasma of stellar atmospheres,but useful also for the diagnostics of laboratory plasmas, investigation of laser produced and inertial fusion plasma as well as for plasma technologies.

We want to draw attention that STARK-B database is one of the databases which enter also in the Virtual Atomic and Molecular Data Center - VAMDC (?Rixon et al., 2011; Dubernet et al., 2016), created in order to enable an efficacious search and mining of atomic and molecular data. scattered in different databases and to make more convenient their adequate use. VAMDC portal with 30 databases with atomic and molecular data, including STARK$\mathrm{B}$, is on the web site: http://portal.vamdc.org/.

\section{CONCLUSION}

Within the frame of MSE method we have calculated Stark widths for six Sc IV spectral lines broadened by collisions with electrons. The obtained data are used to check similarities of Stark widths within a multiplet, supermultiplet and transition array. Also the Stark widths for six Sc IV spectral lines will be implemented in STARK-B database. Other theoretical as well as experimental data for Stark broadening of Sc IV spectral lines do not exist, so that we hope that the obtained results will be of interest, for various problems, especially in stellar physics and laboratory plasma diagnostics.

\section{ACKNOWLEDGEMENT}

This work is a part of the project 176002 "Influence of collisional processes on astrophysical plasma line shapes" supported by the Ministry of Education, Science and Technological Development of Serbia.

\section{REFERENCES}

Adelman, S.J., \& Pintaldo, O. 2000. Elemental abundance analyses with Complejo Astronomico El Leoncito REOSC echelle spectrograms. IV. Extensions of nine previous analyses. Astronomy and Astrophysics, 354, pp. 899-903.

Adelman, S.J., Snow, T.P., Wood, E.L., Ivans, I.I., Sneden, C., Ehrenfreund, P., \& Foing, B.H. 2001. An elemental abundance analysis of the mercury manganese star HD 29647. Monthly Notices of the Royal Astronomical Society, 328(4), pp. 11441150.

Bates, D.R., \& Damgaard, A. 1949. The Calculation of the Absolute Strengths of Spectral Lines. Philosophical Transactions of the Royal Society of London. Series A. Mathematical and Physical Sciences, 242(842), pp. 101-122.

Beauchamp, A., Wesemael, F., \& Bergeron, P. 1997. Spectroscopic Studies of DB White Dwarfs: Improved Stark Profiles for Optical Transitions of Neutral Helium. Astrophysical Journal Supplement, 108, pp. 559-573.
Csillag, L., \& Dimitrijević, M.S. 2004. On the Stark broadening of the $537.8 \mathrm{~nm}$ and $441.6 \mathrm{~nm} \mathrm{Cd}+$ lines excited in a hollow cathode laser discharge. Applied Physics B: Lasers and Optics, 78(2), pp.221-223. doi:10.1007/s00340-003-1368-3

Dimitrijević, M.S. 2003. Stark broadening in Astrophysics: Applications of Belgrade School Results and collaboration of former Soviet republics. Astronomical and Astrophysical Transactions. 22, pp. 389-412.

Dimitrijević, M.S., \& Konjević, N. 1980. Stark widths of doublyand triply-ionized atom lines. Journal of Quantitative Spectroscopy \& amp: Radiative Transfer, 24, pp. 451-459.

Dimitrijević, M.S., \& Kršljanin, V. 1986. Electron-impact shifts of ion lines: Modified semiempirical approach. Astronomy and Astrophysics, 165, pp. 269-274.

Dimitrijević, M.S., \& Popović, L. Č. 2001. Modified Semiempirical Method. Journal of Applied Spectroscopy, 68(6), pp. 893901. doi:10.1023/A:1014396826047

Dimitrijević, M.S., \& Sahal-Bréchot, S. 1992. Stark broadening of spectral lines of multicharged ions of astrophysical interest. V. Sc III and Ti IV lines. Astronomy and Astrophysics Supplement Series, 95, pp. 121-128.

Dimitrijević, M.S., \& Sahal-Bréchot, S. 1998a. Stark broadening of spectral lines of multicharged ions of astrophysical interest. XXI. Sc X, Sc XI, Ti XI and Ti XII spectral lines. Astronomy and Astrophysics Supplement Series, 131, pp. 143-144.

Dimitrijević, M.S., \& Sahal-Bréchot, S. 1998b. Stark broadening parameter tables for Sc X, Sc XI, Ti XI and Ti XII. Serbian Astronomical Journal, 157, pp. 39-64.

Dimitrijević, M.S., \& Sahal-Bréchot, S. 2014. On the Application of Stark Broadening Data Determined with a Semiclassical Perturbation Approach, Atoms, 2, pp. 357-377.

Dubernet, M.L., Antony, B.K., Ba, Y.A., \& et al., 2016. The virtual atomic and molecular data centre(VAMDC) consortium. Journal of Physics B: Atomic, Molecular and Optical Physics, 49(7), p.074003.

Dufour, P., Ben Nessib, N., Sahal-Bréchot, S., Dimitrijević, M.S. 2011. Stark Broadening of Carbon and Oxygen Lines in Hot DQ White Dwarf Stars: Recent Results and Applications. Baltic Astronomy, 20, pp. 511-515.

Elabidi, H., \& Sahal-Bréchot, S. 2011. Checking the dependence on the upper level ionization potential of electron impact widths using quantum calculations. European Physical Journal D, 61, pp. 285-290.

Gornushkin, I.B., King, L.A., Smith, B.W., Omenetto, N., \& Winefordner, J.D. 1999. Line broadening mechanisms in the low pressure laser-induced plasma. Spectrochimica Acta, Part B: Atomic Spectroscopy, 54(8), pp. 1207-1217.

Griem, H.R. 1968. Semiempirical Formulas for the ElectronImpact Widths and Shifts of Isolated Ion Lines in Plasmas. Physical Review, 165(1), pp. 258-266.

Griem, H.R. 1974. Spectral line broadening by plasmas. New York: Academic Press, Inc. 
Griem, H.R. 1992. Plasma spectroscopy in inertial confinement fusion and soft x-ray laser research. Physics of Fluids, 4(7), pp. 2346-2361.

Kahraman Alicavus, F., Niemczura, E., Polińska, M., Helminiak, K.G., Lampens, P., Molenda-Żakowicz, J.,... Kambe, E. 2017. High-resolution spectroscopy and abundance analysis of $\delta$ Scuti stars near the $\gamma$ Doradus instability strip. Monthly Notices of the Royal Astronomical Society, 470(4), pp. 4408-4420.

Keane, C.J., Lee, R.W., Hammel, B.A., Osterheld, A.L., Suter, L.J., Calisti, A.,... Talin, B. 1990. Line broadening of Ne-like xenon as a diagnostic for high-density implosion experiments. Review of Scientific Instruments, 61(10), pp. 2780-2782.

Konjević, N. 1999. Plasma broadening and shifting of nonhydrogenic spectral lines: present status and applications. Physics Reports, 316(6), pp. 339-401.

Kramida, A., Ralchenko, Yu., Reader, J.,\& -NIST ASD Team. 2017. NIST Atomic Spectra Database. Gaithersburg, MD: National Institute of Standards and Technology.(ver. 5.5.1), Retrived from https://physics.nist.gov/asd 2017, Nov 18.

Milovanović, N., Dimitrijević, M.S., Popović, L.Č., \& Simić, Z. 2004. Importance of collisions with charged particles for stellar UV line shapes: Cd III. Astronomy and Astrophysics, 417, pp. 375-380.

Oertel, G.K., \& Shomo, L.P. 1968. Tables for the Calculation of Radial Multipole Matrix Elements by the Coulomb Approximation. Astrophysical Journal Supplement, 16, pp. 175-218.

Peláez, R.J., Ćirišan, M., Djurović, S., Aparicio, J.A., Mar, S. 2009. Stark broadening measurements of low-intensity singly and doubly ionized xenon spectral lines. Astronomy and Astrophysics, 507(3), pp. 1697-1705.

Popović, L.Č., \& Dimitrijević, M.S. 1996. Stark widths for astrophysically important ns-np transitions in Sc II, Y II and Zr II spectra. Astronomy and Astrophysics, 120, pp. 373-374. Supplement Series

Popović, L.Č., \& Dimitrijević, M.S. 1997. Stark broadening parameter tables for Xe II, Sc II, Y II and Zr II. Bulletin Astronomique de Belgrade, 155, pp. 159-163.

Popović, L.Č., Simić, S., Milovanović, N., Dimitrijević, M.S. 2001. Stark Broadening Effect in Stellar Atmospheres: Nd II Lines. Astrophysical Journal, 135(1), pp. 109-114. Supplement Series. doi:10.1086/321778

Rauch, T., Ziegler, M., Werner, K., \& et al., 2007. High-resolution FUSE and HST ultraviolet spectroscopy of the white dwarf central star of Sh 2-216. Astronomy and Astrophysics, 470(1), pp. 317-329.

Richou J., \& Molitor A. 1970. Determination of the electron density of a xenon plasma, created in a shock tube, by laser interferometry and spectroscopy. Comptes Rendus de L'Académie de Science, Serie B, 271, pp. 753-756.

Rixon, G., Dubernet, M.L., Piskunov, N., \& et al., 2011. VAMDCThe Virtual Atomic and Molecular Data Centre: A New Way to Disseminate Atomic and Molecular Data-VAMDC Level 1 Release. . In: AIP Conference Proceedings., pp. 107-115 1344.
Sahal-Bréchot, S., Dimitrijević, M.S., \& Moreau, N. 2017. STARK-B database. Observatory of Paris / LERMA and Astronomical Observatory of Belgrade. Retrieved from http://starkb.obspm.fr 2017 November 12.

Sahal-Bréchot, S., Dimitrijević, M.S., Moreau, N., \& Ben Nessib, N. 2015. The STARK-B database VAMDC node: a repository for spectral line broadening and shifts due to collisions with charged particles. Physica Scripta, 50, 054008.

Seidel, S., Wrubel, Th., Roston, G., \& Kunze, H.-J. 2001. Line profile measurements of $\left({ }^{4} S\right) 6 s^{5} S-\left({ }^{4} S\right) 6 p^{5} \mathrm{P}$ transitions of Xe III. Journal of Quantitative Spectroscopy and Radiative Transfer, 71(2-6), pp. 703-709.

Simić, Z., Dimitrijević, M.S., \& Kovačević, A. 2009. Stark broadening of spectral lines in chemically peculiar stars: Te I lines and recent calculations for trace elements. New Astronomy Review, 53(7-10), pp. 246-251.

Simić, Z., Dimitrijević, M.S., Milovanović, N., \& Sahal-Bréchot, S. 2005a. Stark broadening of Cd I spectral lines. Astronomy and Astrophysics, 441(1), pp. 391-393.

Simić, Z., Dimitrijević, M.S., Popović, L.Č., \& Dačić, M. 2005 b. Stark Broadening of F III Lines in Laboratory and Stellar Plasma. Journal of Applied Spectroscopy, 72(3), pp. 443-446. doi:10.1007/s10812-005-0095-4

Simić, Z., Dimitrijević, M.S., Popović, L.Č., \& Dačić, M. 2006. Stark broadening parameters for Cu III, Zn III and Se III lines in laboratory and stellar plasma. New Astronomy, 12(3), pp. 187191.

Sorge, S., Wierling, A., Röpke, G., Theobald, W., Suerbrey, R., \& Wilhein, T. 2000. Diagnostics of a laser-induced dense plasma by hydrogen-like carbon spectra. Journal of Physics B: Atomic, Molecular and Optical Physics, 33(16), pp. 2983-3000.

Sugar, J., \& Corliss, C. 2004. Atomic Energy Levels of the IronPeriod Elements: Potassium through Nickel. Journal of Physical and Chemical Reference Data, 14, pp. 1-664, Supplement 2.

Tankosić, D., Popović, L.Č., \& Dimitrijević, M.S. 2003. The electron-impact broadening parameters for Co III spectral lines. Astronomy and Astrophysics, 399, pp. 795-797.

Torres, J., van de Sande, M.J., van der Mullen, J.J.A.M., Gamero, A., \& Sola, A. 2006. Stark broadening for simultaneous diagnostics of the electron density and temperature in atmospheric microwave discharges. Spectrochimica Acta, Part B: Atomic Spectroscopy, 61(1), pp. 58-68.

van Regemorter, H., Hoang Binh, D., \& Prud'homme, M. 1979. Radial transition integrals involving low or high effective quantum numbers in the Coulomb approximation. Journal of Physics B: Atomic, Molecular and Optical Physics, 12, pp. 1053-1061. Wiese, W.L.,\& Konjević, N. 1982. Regularities and similarities in plasma broadened spectral line widths(Stark widths). Journal of Quantitative Spectroscopy and Radiative Transfer, 28, pp. 185198.

Wieser, J., Murnick, D.E., Ulrich, A., Huggins, H.A., Liddle, A., Brown, W.L. 1997. Vacuum ultraviolet rare gas excimer light source. Review of Scientific Instruments, 68(3), pp. 1360-1364. 